\title{
On the Importance of 3D, Geometrically Accurate, and Subject-Specific Finite Element Analysis for Evaluation of in-Vivo Soft Tissue Loads
}

\author{
Kevin M. Moerman ${ }^{a, b^{*}}$, Marc van Vijven ${ }^{d}$, Leandro R. Solis ${ }^{c}$, Eline E. van \\ Haaften ${ }^{d}$, Arjan C.Y. Loenen ${ }^{d}$, Vivian K. Mushahwarc, Cees W.J. Oomens ${ }^{d}$ \\ a Media-Lab., Centre for Extreme Bionics, Massachusetts Institute of Technology, Cambridge, MA, United States of America \\ b Trinity Centre for Bioengineering, University of Dublin, Trinity College, Dublin, Ireland \\ c Faculty of Medicine and Dentistry, University of Alberta, Edmonton, Canada \\ d Biomedical Engineering Department, Eindhoven University of Technology, The Netherlands
}

\begin{abstract}
Pressure ulcers are a type of local soft tissue injury due to sustained mechanical loading and remain a common issue in patient care. People with spinal cord injury (SCl) are especially at risk of pressure ulcers due to impaired mobility and sensory perception. The development of load improving support structures relies on realistic tissue load evaluation e.g. using finite element analysis (FEA). FEA requires realistic subject-specific mechanical properties and geometries. This study focuses on the effect of geometry. MRI is used for the creation of geometrically accurate models of the human buttock for three able-bodied volunteers and three volunteers with $\mathrm{SCl}$. The effect of geometry on observed internal tissue deformations for each subject is studied by comparing FEA findings for equivalent loading conditions. The large variations found between subjects confirms the importance of subject-specific FEA.
\end{abstract}

Keywords:

Finite Element Analysis, Image-based modelling, geometry, soft tissue, pressure ulcer

*Corresponding author:

K.M. Moerman

ORCID iD 0000-0003-3768-4269

MIT Media Lab

75 Amherst Street

Cambridge, MA 02139, USA.

Telephone: +17813548683

Email:kmoerman@mit.edu 


\section{Introduction}

Pressure ulcers are a form of localized soft tissue injury to the skin and/or underlying tissue, usually over a bony prominence, resulting from sustained mechanical loading, such as pressure and shear deformations [20]. Some pressure ulcers develop near the skin surface and may extend to deeper layers if unattended. However, there is a category that starts in deeper soft tissue layers adjacent to bony prominences. The latter category is referred to as deep tissue injury (DTI). Persons with reduced mobility and impaired sensory perception - including people with spinal cord injury $(\mathrm{SCl})$ - are especially at risk of developing pressure ulcers such as DTI, because they are unable to detect damage development and actively adjust their posture to redistribute the loading themselves [3]. Pressure ulcers form an enormous financial burden for healthcare systems worldwide. For example, in the United States, the annual cost of treating pressure ulcers that develop during a hospital stay is estimated to exceed \$11 billion [29]. Pressure ulcers can be painful, depressing [4] and are associated with fatal septic infections and tens of thousands of deaths each year in the United States [22].

A large number of studies that focused on skeletal muscle cells and other soft tissues, have confirmed that there are two damage mechanisms in the development of DTI: prolonged mechanical deformation and reduced oxygenation due to the occlusion of blood vessels [21]. The first damage mechanism involves direct deformation damage at a threshold level, which has been estimated to be between 0.5 and 0.6 [12] for the maximum shear strain.

In order to study the mechanisms behind pressure ulcer development, and to develop and evaluate management and prevention techniques, detailed knowledge of invivo soft tissue loading is required. This includes the soft tissue stress and deformation distribution. These are however challenging to assess experimentally. Computational modelling techniques, such as finite element analysis (FEA), on the other hand, do provide information on the internal mechanical state throughout the entire tissue. Hence accurate 
FEA plays an important role in the understanding of the mechanisms of pressure ulcer formation (e.g. [12] [10][8]). In addition, they are vital tools for FEA based design and development of technologies and support systems that can reduce the incidence of pressure ulcers (e.g. [7][9]).

However, FEA requires detailed knowledge on the subject-specific biomechanical properties, boundary conditions, and an accurate description of the geometry. In terms of FEA geometries, 2D models are common (e.g. [7]), and 3D models have also been explored (e.g. [9]). To date these studies have confined analysis to FEA of single subjects, and direct FEA comparisons between multiple subjects or able-bodied volunteers and patient populations are lacking. It is therefore unclear if findings concerning tissue loading from single individuals can be generalised to other individuals, or if findings from able-bodied subjects are descriptive for people with $\mathrm{SCl}$. This study therefore investigates the importance of 3D and subject-specific geometry in FEA outcomes of loading to the human buttock. Magnetic resonance images (MRI) of the buttocks region were obtained from three able-bodied (AB) volunteers and three volunteers with $\mathrm{SCl}$, allowing for detailed FEA model construction. The models were each assigned the same soft tissue mechanical properties, and were subjected to equivalent plate compression loading at levels of up to $240 \mathrm{~N}$ (35$45 \%$ of body weight). Internal tissue deformations predicted by FEA were then compared for each subject. Using these data, the following research questions were addressed: 1) To what extent will predicted loading vary across subjects? 2) To what extent does the predicted loading vary between the $\mathrm{AB}$ and $\mathrm{SCl}$ groups?

\section{Methods}

\section{MRI based derivation of subject-specific geometry}

Subject-specific geometries were obtained from MRI data for three able-bodied volunteers and three volunteers with SCI (Table 1). All experimental procedures were approved by the University of Alberta Research Ethics Board, and informed consent was obtained prior to the initiation of data acquisition. MRI scans were acquired using a 1.5T scanner (Siemens 
Sonata, Siemens Medical Systems, Erlangen, Germany) at the Peter S. Allen MR Research Centre at the University of Alberta.

Table 1: Subject parameters
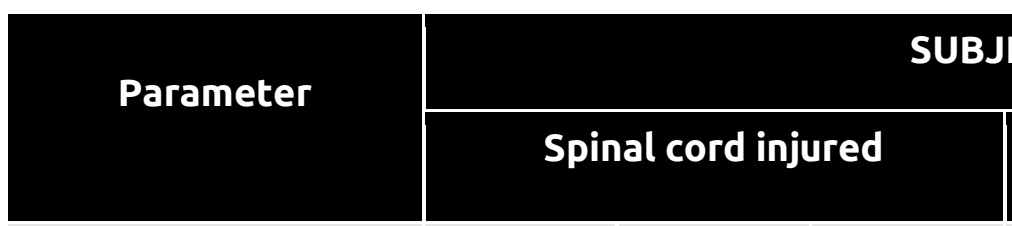

Participant ID\#

Gender

Age (years)

Weight (Kg)

Years since injury

Injury level
1

M

30

63.5

10

C5-6
2

F

45

54.4

16

T4-5
3

M

55

56.7

2

C7

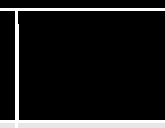

1

M

27

70.3

N/A

N/A
N/A

Able bodied

2 3

F

24

63.5

68.0

N/A

N/A

Subjects were positioned in a supine position with their legs placed over a leg support to avoid loading the buttock region from making contact with the MRI bed (Figure 1). All imaging was performed using a RF body coil in combination with a spine array. The field of view (FOV) captured the anatomy of the buttock, pelvic region and upper thigh (T1, proton density, and gradient recalled echo data, acquisition matrix 512x416, 60 slices, FOV $400 \times 325 \times 240 \mathrm{~mm}$, voxel size $0.78 \times 0.78 \times 4.00 \mathrm{~mm}$ ).

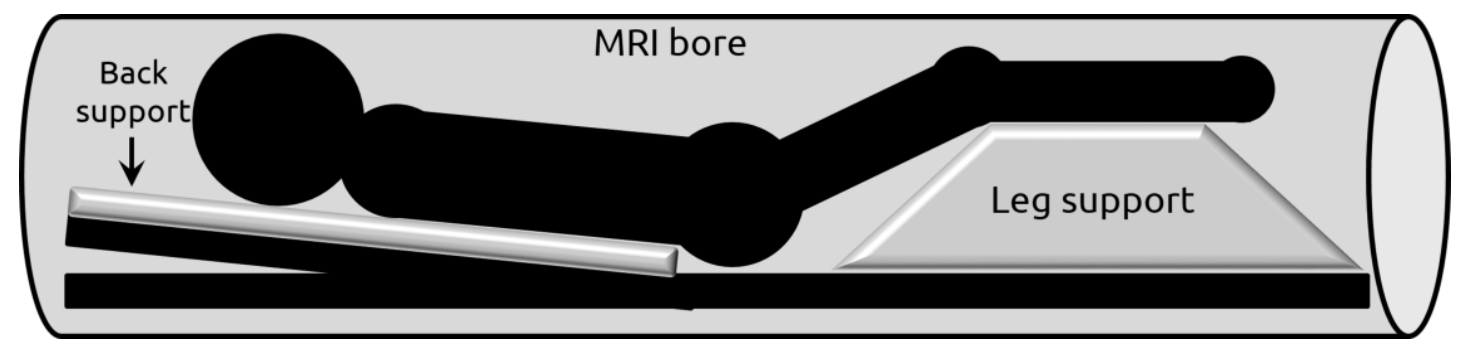

Figure 1 Schematic overview of subject positioning in the MRI bore showing back and leg support systems.

Skin, adipose-muscle tissue and bone boundaries were manually segmented from the MRI data using the software Mimics (v14.0, Materialise, Leuven, Belgium). All bone tissue was jointly segmented. In some images the skin contour on the left and right of the MRI data 
was not fully captured in the FOV for all slices (Figure 2A). For these slices the contour was manually completed (see added parts on left and right in Figure 2B). Segmentations (Figure 2B) were imported into MATLAB (R2015a The Mathworks Inc., Natick, MA) and processed using the open source GIBBON toolbox (r89, $[15,16])$. The segmentations were converted to triangulated surface models (Figure 2C 1-3) with a desired point spacing and smoothened (shrinkage avoiding smoothening algorithm [27]). Through Boolean operations on the set of meshes a single closed, multi-boundary surface model was created consisting of the outer skin surface, an adipose-muscle boundary surface, and a joint bone surface (Figure 2C 4).

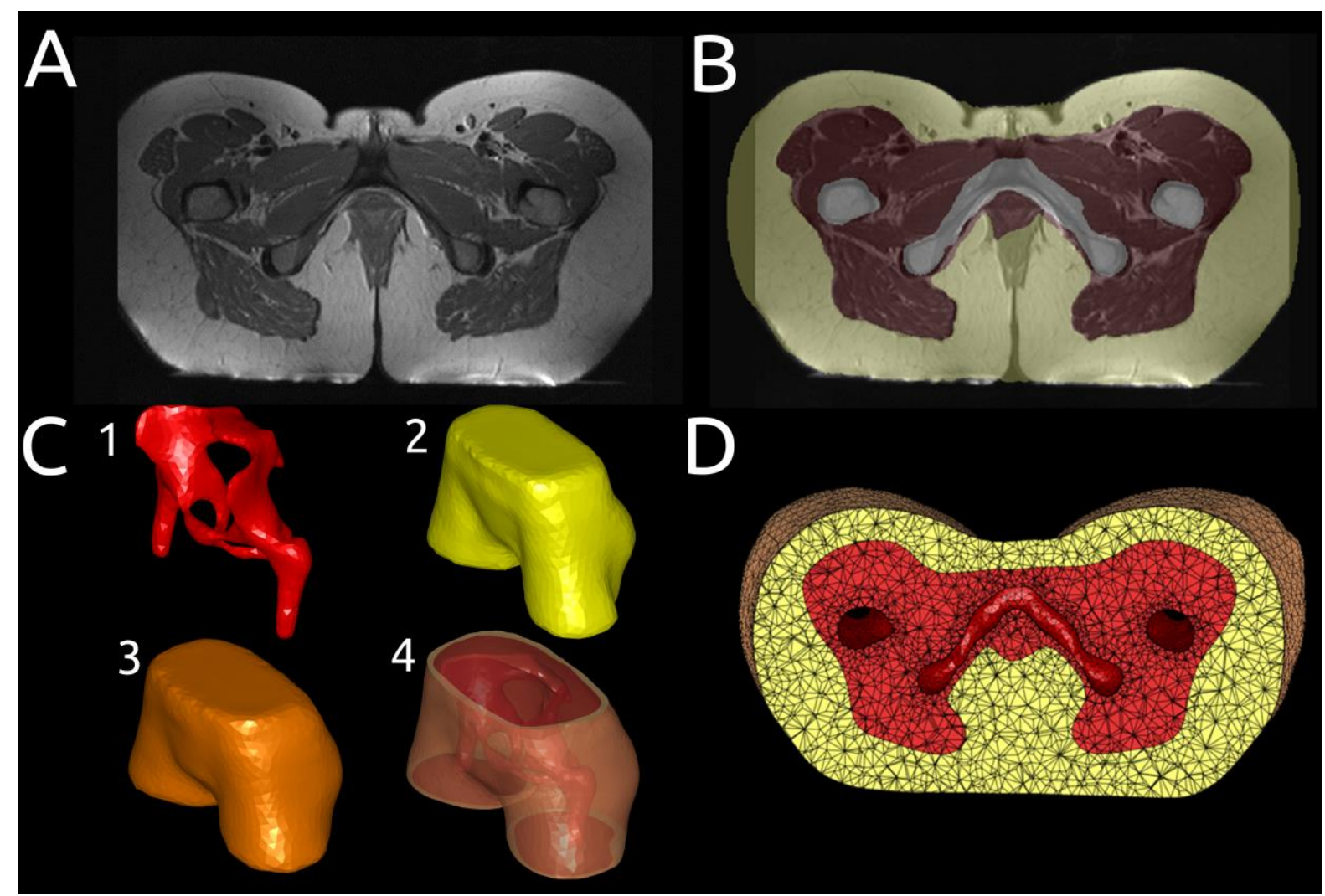

Figure 2: An example MRI slice (A), a tissue segmentation overlaid (B), the derived surface geometry (C) for the bones (C1), muscle-adipose tissue boundary (C2), and the skin (C3), which can be combined to create a closed surface geometry (C4), suitable for solid meshing for FEA, see cut-view (D)

\section{FEA model construction}

Following the extraction of the subject surface geometries, the 3D meshing, boundary condition specification and FEA procedures were coded and automated using the GIBBON toolbox, which contains the open source meshing software TetGen (v1.5.0, [23]) used for 
tetrahedral meshing, and employs FEBio (v2.1.1, [14], Musculoskeletal Research Laboratories, The University of Utah, USA) for FEA. Coding FEA procedures ensures repeatable and consistent analysis across all subjects.

\section{Solid and surface meshing}

All models were assigned two solid material regions, an adipose region and a muscle region. The latter was termed muscle tissue here, but effectively jointly represented all soft tissues between the muscle-adipose boundary and the bones. The solid regions were meshed using tri-linear tetrahedral elements (Figure 2D). The mesh near the plate was locally refined. After the solid adipose and muscle tissue regions were meshed, a skin shell element layer was specified coinciding with the triangular elements of the outer tissue surface. The thickness of the shell elements was set to $1 \mathrm{~mm}$, in agreement with thicknesses employed elsewhere $[5,6,17,18]$. The final FEA meshes consisted of approximately 100000 solid tetrahedral elements and 7000 shell elements for the skin layer. For compression a rigid flat plate was simulated and meshed using rigid triangular shell elements (Figure 3).
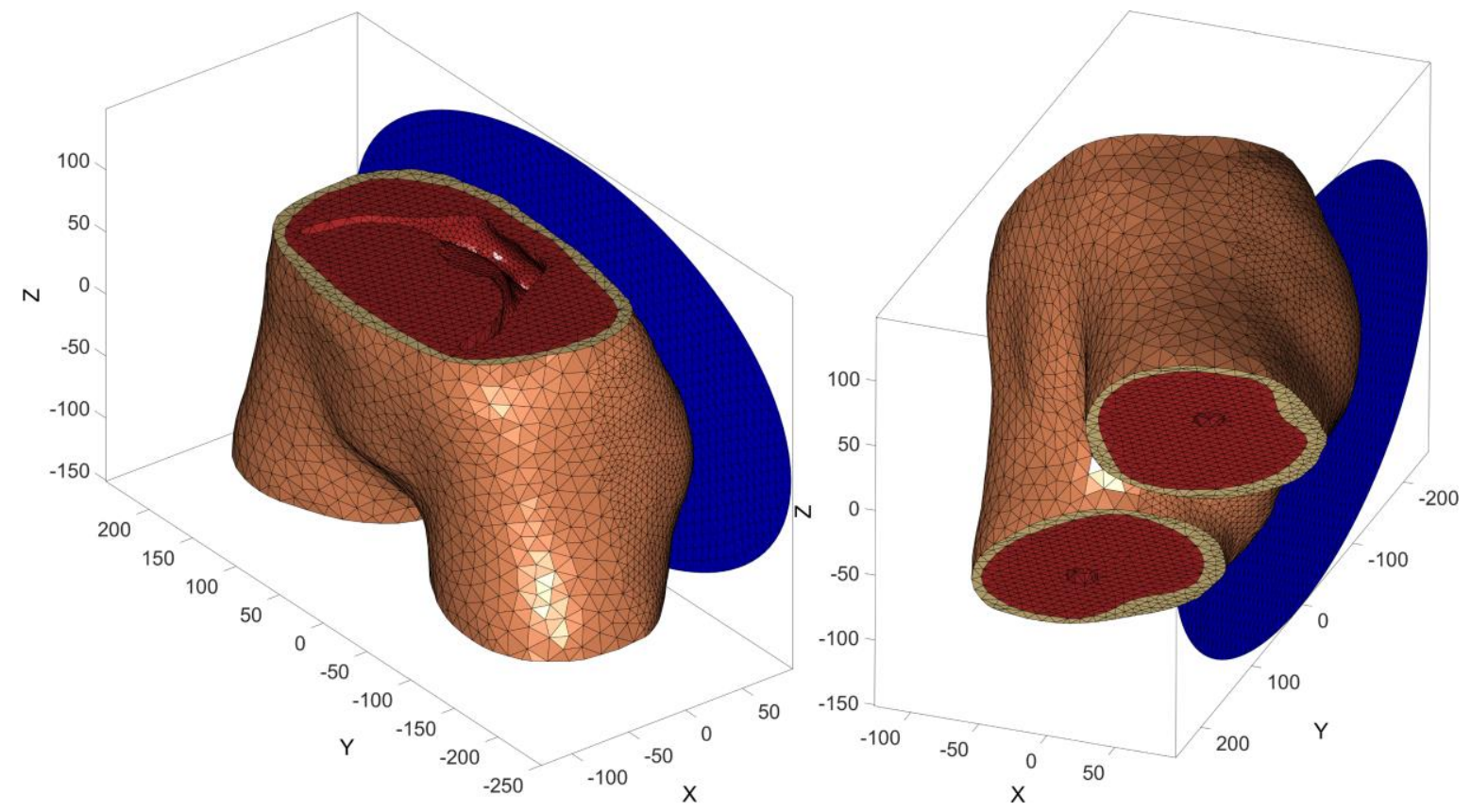

Figure 3: Two visualizations of a model showing the plate used for compression in blue 


\section{Constitutive modelling}

Three deformable tissue types were distinguished: skin, adipose, and muscle tissue. The material behaviour of each tissue type was modelled using the following uncoupled strain energy density formulation (a first order Ogden form [24]):

$$
\Psi=\frac{c}{m^{2}}\left(\tilde{\lambda}_{1}^{m}+\tilde{\lambda}_{2}^{m}+\tilde{\lambda}_{3}^{m}-3\right)+\frac{\kappa}{2} \ln (J)^{2}
$$

where $\tilde{\lambda}_{i}$ are the deviatoric principal stretches, and $c$ and $m$ are deviatoric material parameters. The deviatoric response is linearly scaled by $c$ while the degree of non-linearity is controlled by $m$. The volumetric behaviour is dictated by the material bulk-modulus $\kappa$. Due to its high water content soft tissue is often assumed to be nearly incompressible, such that $J=\operatorname{det}(\mathbf{F}) \approx 1$. In the current study $\kappa=400 * c$ for all simulations, leading to $0.99<J<1.01$. The remaining constitutive parameters employed are shown in Table 2.

Table 2: Constitutive parameters

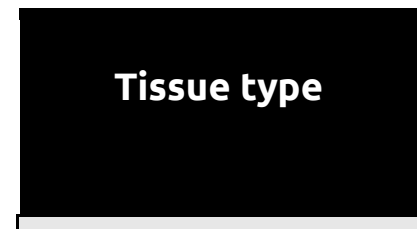

Skin

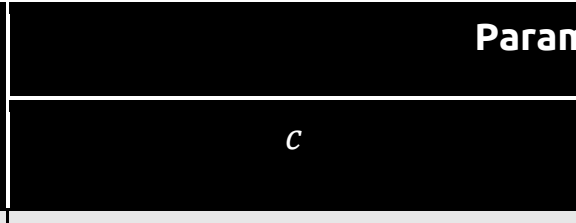

49.7 $\mathrm{MPa}$ $m$

2

2

12

Because the focus of the study was on the effects of subject-geometry independent of material parameters, all subjects were assigned the same mechanical properties. For adipose tissue, the parameters stemmed from the work by [25]. For skin and muscle tissue, the parameters were manually adjusted to obtain qualitatively realistic contact pressures and deformation characteristics. The final $c$ and $m$ values for skin and muscle were consistent with ranges presented in the literature [19] [2]. In addition, for muscle tissue the $c$ and $m$ values produce behaviour in close agreement with [13]. 


\section{FEA boundary conditions}

In order to compare equivalent levels of loading for each subject, compressions with a rigid plate (Figure 3) were simulated to forces up to $240 \mathrm{~N}$. This represented between $35-45 \%$ of body weight depending on the subject. Displacement control was used for plate motion, which was iteratively adjusted to reach the final target force to within $2 \mathrm{~N}$. A zero-friction sliding interface was defined between the rigid plate (master) and skin (slave) surface. Bones were represented as rigid voids by fully supporting bone surface nodes.

\section{Comparative analysis of effect of subject geometry}

Variation of loading conditions within a group (able bodied and $\mathrm{SCl}$ ) and across groups was investigated to assess the effect of geometry on internal tissue loading. The comparison focussed on analysis of: 1) indentation distance, 2) maximum shear strains, and 4) tissue volume subjected to maximum shear strains higher than 0.5 . The maximum shear strain is defined as:

$$
E_{\max }=\frac{1}{2} \max \left(\left|E_{1}-E_{2}\right| \quad\left|E_{2}-E_{3}\right| \quad\left|E_{3}-E_{1}\right|\right)
$$

where $E_{i}$ are the eigenvalues of the Green-Lagrange strain tensor. Shear strain levels of 0.5 were identified as the threshold for tissue injury [12]; therefore, the total tissue volume subjected to $E_{\max }>0.5$ was identified in this study as the damage risk volume (DRV).

\section{Results}

\section{Subject-specific 3D FEA}

For the current study six MRI derived subject specific 3D FEA models were constructed. Apart from segmentation, the subject geometry creation, meshing, boundary condition specification and FEA were all achieved using freely available, and open source modelling tools. The framework, based on the GIBBON toolbox, allows for code based FEA. By coding the model generation, repeatable and consistent meshing and boundary condition specification was achieved across all subjects. Models typically consisted of 100000 solid, and 7000 shell elements, and the computational time was typically 60 min (Intel Core i74910MQ CPU, 32Gb RAM). 
Compressive plate boundary conditions

Table 3 lists the applied plate displacements and the resulting plate reaction forces for each subject. In all cases, the target force was reached to within $2 \mathrm{~N}$. The final plate displacements occurred in the ranges $14.8-33.1 \mathrm{~mm}$, and $24.7-35.2 \mathrm{~mm}$ for the $\mathrm{SCl}$ and $\mathrm{AB}$ groups, respectively. A large degree of variation is observed within the $\mathrm{SCl}$ group with $\mathrm{SCl}$ subject 1 presenting with nearly double the place displacement of that of SCl subjects 2 and 3. The variation of plate displacements appears less pronounced for the able bodied volunteers which present on average with $9.5 \mathrm{~mm}$ more plate displacement than the average of the three $\mathrm{SCl}$ subjects.

Table 3: Applied force boundary conditions

\begin{tabular}{|c|c|c|c|c|c|c|c|c|}
\hline \multirow{3}{*}{$\begin{array}{c}\text { Parameter } \\
\text { Participan } \\
\text { t ID\# }\end{array}$} & \multicolumn{8}{|c|}{ SUBJECTS } \\
\hline & \multicolumn{4}{|c|}{ Spinal cord injured } & \multicolumn{4}{|c|}{ Able bodied } \\
\hline & 1 & 2 & 3 & Mean & 1 & 2 & 3 & Mean \\
\hline $\begin{array}{l}\text { Final plate } \\
\text { force }(\mathrm{N})\end{array}$ & 241 & 243 & 241 & 242 & 243 & 243 & 242 & 243 \\
\hline $\begin{array}{c}\text { Final plate } \\
\text { displacemen } \\
\text { t (mm) }\end{array}$ & 33.1 & 14.8 & 17.6 & 21.8 & 35.2 & 24.7 & 34.0 & 31.3 \\
\hline
\end{tabular}

\section{Internal tissue deformations}

Figure 4 visualizes the maximum shear strains found in the mid sagittal plane for each subject at a force of 50 and $240 \mathrm{~N}$. For all subjects the highest strains present around the coccyx. Maximum shear strains in excess of 0.5 are observed for all subjects at $240 \mathrm{~N}$, but only for $\mathrm{SCl}$ subject 1 and 2 at $50 \mathrm{~N}$. At the $240 \mathrm{~N}$ load, high maximum shear strains spread distally of the coccyx for $\mathrm{SCl}$ subject 1 and $\mathrm{AB}$ subject 1 and 3 . However, high maximum shear strains remain more localized at the coccyx for $\mathrm{SCl}$ subjects 2 and 3, and $\mathrm{AB}$ subject 2 . 


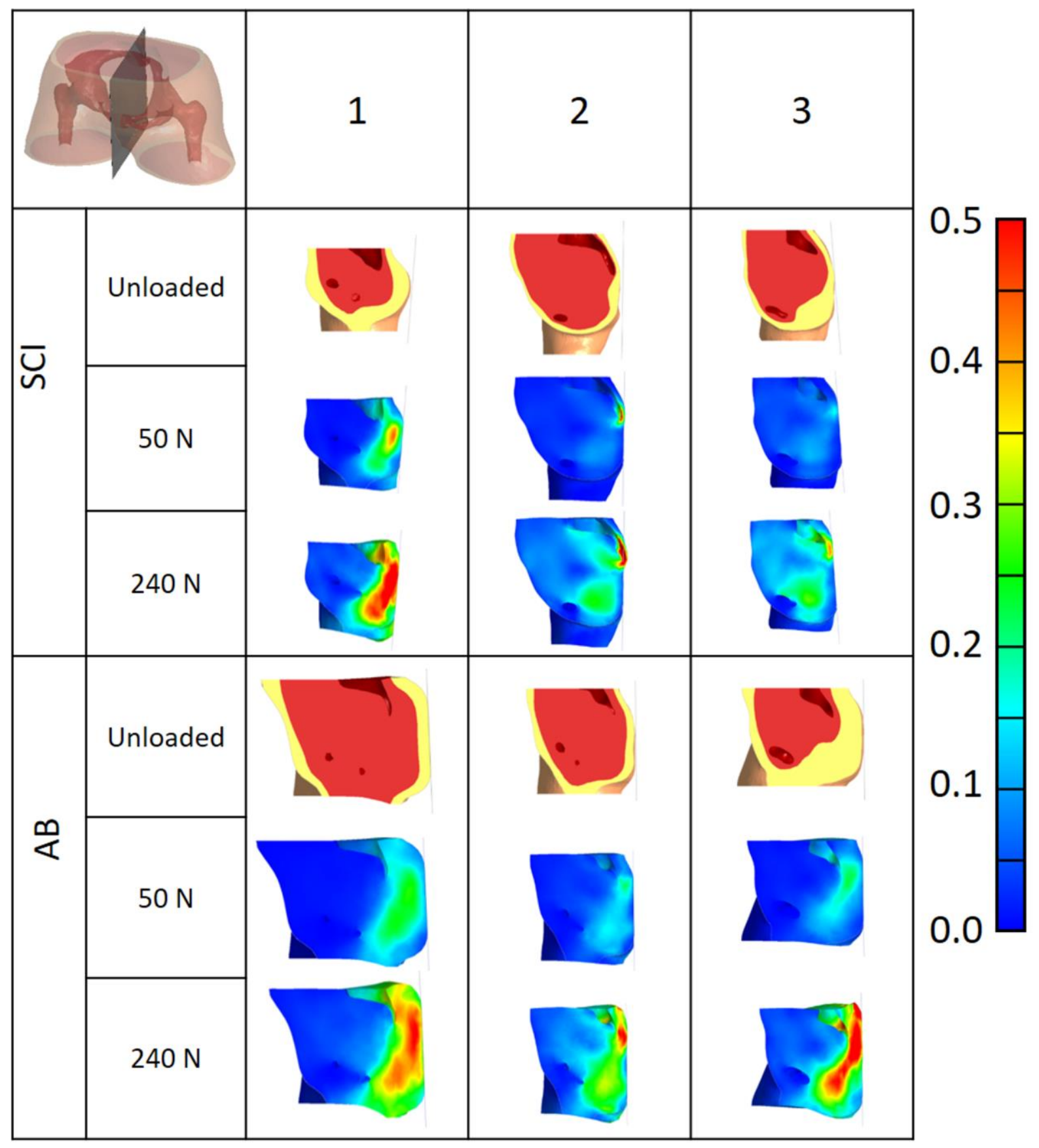

Figure 4: Maximum shear strain results for $50 \mathrm{~N}$ (middle rows), $240 \mathrm{~N}$ (bottom rows). A schematic for the central location of the cut plane is shown in the top left image.

To further investigate the extent of potentially damaging strains, Figure 5 and Figure 6 present DRV data as a function of loading for each subject. In Figure 5 the total DRV is shown as a function of force (Figure 5A) or force percentage relative to body weight (Figure 5B). These data illustrate the large degree of variation between subjects. As with the plate displacements and maximum shear strain patterns, $\mathrm{SCl}$ subject 1 presents with a different behaviour when compared to $\mathrm{SCl}$ subject 2 and 3 which are relatively similar. The $A B$ 
subjects presented with less variation and present with higher DRV levels than $\mathrm{SCl}$ subjects 1 and 2 but lower levels than SCI subject 1 .
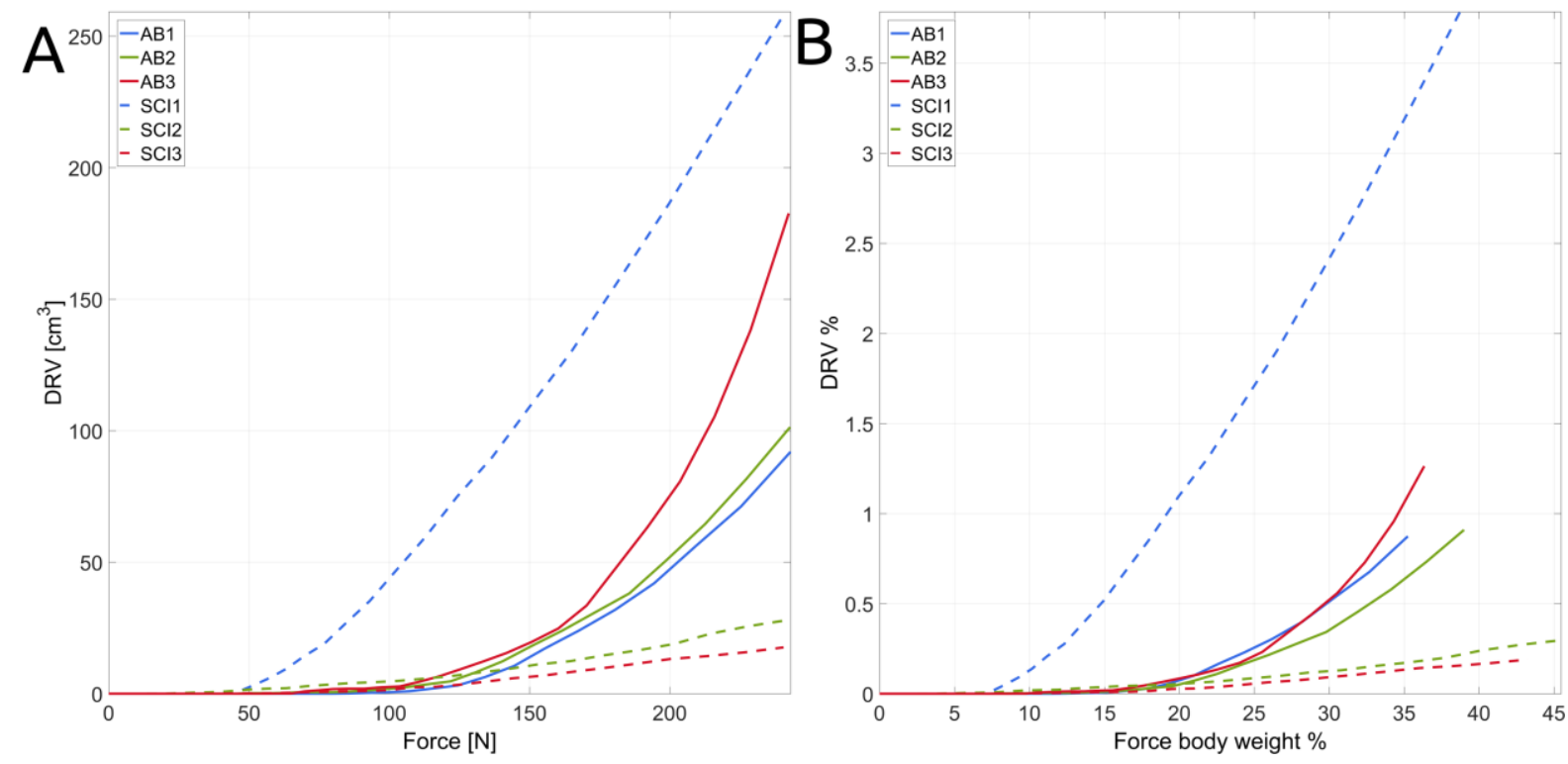

Figure 5 Loading force against DRV for each subject (A), and the force percentage (with respect to body weight) against DRV percentage (with respect to total volume) for each subject (B).

In Figure 6 relative DRV percentages (with respect to total tissue model volume) are plotted against force percentages (with respect to body weight) for each subject. In addition, Figure 6 presents results not only for the total tissue but also the adipose and muscle tissue independently. The results show that although the overall response in terms of DRV is similar for the AB subjects, the loading is distributed across the tissue types differently, i.e., for $A B$ subject 1 and $A B$ subject 2 the $D R V$ is predominantly found in muscle tissue, an effect not observed as dominantly for the other subjects. For the volunteers with $\mathrm{SCl}$, subjects $\mathrm{SCl}$ 2 and $\mathrm{SCl} 3$ showed a similar response. However, $\mathrm{SCl} 1$ showed the highest DRV percentage of all subjects for all loads. 

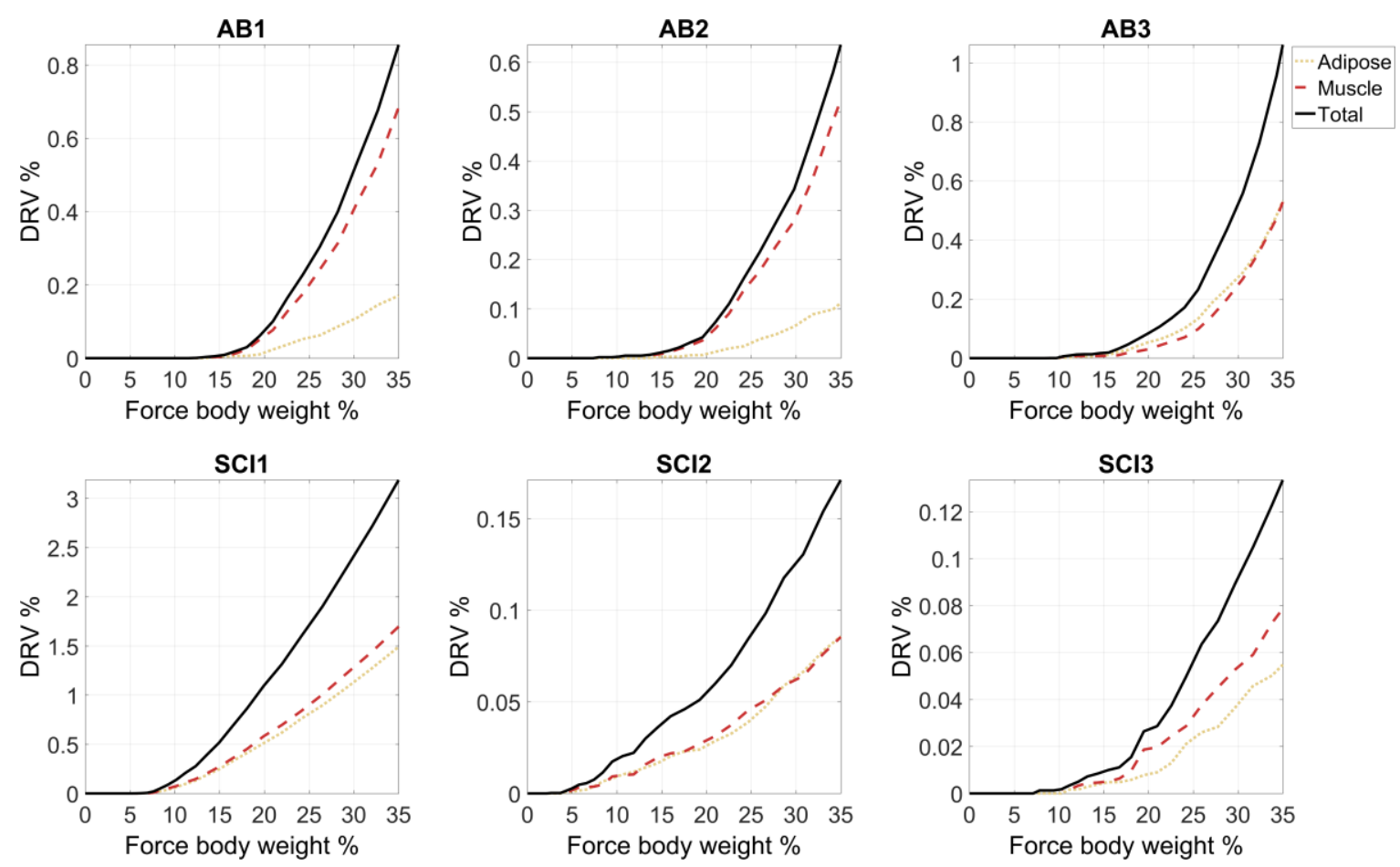

Figure 6 The DRV percentages (with respect to total volume) against force percentage (with respect to body weight). Results are split into those for the total tissue, and the muscle and adipose tissue independently. The horizontal axis was cropped 35\%, a level up to which all subjects have data.

\section{Discussion}

In order to study the effects of geometry on FEA findings for loading in the human buttocks, detailed 3D FEA models were constructed based on subject-specific MRI data. FEA of plate induced loading, similar to lying on a rigid surface, was performed for three able bodied volunteers and three volunteers with $\mathrm{SCl}$. Each of the models was assigned the same mechanical properties and was subjected to the same force (up to $240 \mathrm{~N}$ ) loading by a rigid flat plate.

For the maximum load, plate displacements were found in the ranges $14.8-33.1 \mathrm{~mm}$, and 24.7-35.2 mm for the $\mathrm{SCl}$ and $\mathrm{AB}$ groups, respectively. The smallest displacement of 14.8 mm was for $\mathrm{SCl} 2$ who presents with very little soft tissue covering the coccyx (Figure 4); hence, this subject presented with highly localized and high maximum shear strains at the coccyx and forces increased faster as a function of plate displacement due to the proximity of the bone to the plate. The opposite occurred for $A B 1$, with the highest plate displacement of $35.2 \mathrm{~mm}$, and presented with a thicker layer of tissue at the bony features (Figure 4). As shown in Figure 5, in terms of DRV the AB subjects presented with a very 
similar response. AB 3 however had relatively more adipose tissue and therefore this tissue was more involved when compared to $\mathrm{AB} 1$ and $\mathrm{AB} 2 \mathrm{SCl} 1$ showed the highest, and earliest onset, of DRV. However, interestingly, the other two SCI subjects appeared least vulnerable of all in terms of DRV for the loads evaluated. This illustrates the level of variability in predicted tissue loading due to the varying subject geometries. The main take home message of this study is therefore that internal tissue loading is highly dependent on the subject-specific geometry. Further it was found that based on geometry alone SCl subjects do not necessarily present as more vulnerable than $A B$ subjects.

Few studies have compared tissue deformation characteristics of $A B$ and $\mathrm{SCl}$ subjects. In addition, experimental conditions and analysis methods vary significantly, thus hindering detailed and direct comparison to the literature. Linder-Ganz et al. 2008 [11] studied loading in the buttocks of $A B$ and paraplegic individuals for sitting ( $n=6 A B, n=6 S C l$ ) and lying ( $\mathrm{n}=2 \mathrm{AB}$ and $\mathrm{n}=6 \mathrm{SCl}$ ) by using 2D MRI before and after weight-bearing, combined with 2D subject-specific FEA. On average the group with paraplegia presented with higher stresses and strains and were therefore considered more vulnerable. However, the range of derived maximum shear strains, for both groups and both load cases, did overlap due to subject-to-subject variations, a result consistent with the findings presented here.

Because the current study included only three volunteers in each of the $A B$ and $S C I$ groups, a thorough statistical analysis was not possible. Nevertheless, the findings on variation between subjects are valuable and can be used to formulate recommendations for FEA, i.e., that subject-specific analysis is required.

The DRV data represents the volume of tissue subjected to maximum shear strains in excess of 0.5 (motivated by [12]). As investigations into damage thresholds are still ongoing, other thresholds and damage risk factors may be proposed in the future. Further, thresholds are likely subject-specific. The relative DRV measures presented here were chosen to enable comparison between subjects. Other means to draw comparisons between subjects may be proposed, e.g. to make data relative to volume of a particular tissue region rather than the entire model. In addition, independent of threshold and 
damage risk interpretation, it remains likely that subjects presenting with high strains are most vulnerable. Further the findings of variability hold irrespective of chosen damage risk measure.

In the current study a single set of literature based constitutive parameters was used for all subjects. In reality, these parameters are subject-specific and may depend on pathology. However, since in the current study the effect of geometry alone was of interest, this simplification was deemed justified. Future work featuring subject-specific constitutive properties will aid in the more realistic evaluation of tissue loading and therefore the subject's risk to injury given a particular loading regime. Further, tissue anisotropy should be considered.

In this study, the soft tissue is represented by skin, adipose, and muscle tissue regions. The mechanical behaviour in each region was modelled as homogenous and continuous. In reality these regions may not be homogeneous. The muscle tissue for instance has subject-specific, and spatially varying, levels of intramuscular fat. Intramuscular fat infiltration has been reported for people with $\mathrm{SCl}$ [28] and has been shown to be a risk factor for tissue loading [26]. Therefore, subjects with increased intramuscular fat may be at an increased risk. In the study by Sopher et al. 2011 [26], the effects of intramuscular fat were studied through FEA of loading for the same geometry but with varying degrees of fat infiltration. Conversely in our study, since the effect of 3D geometry was of interest, the muscle tissue properties were the same across all subjects while geometries varied. Future work could investigate the relative importance of either or both geometrical variations and tissue composition variations.

Tissue loading due to external rigid plate induced compression was considered here. For different load cases, the internal tissue loading and the degree of variability between subjects may change. Loading to the buttock typically occurring during sitting or lying is most relevant to pressure ulcer research, and the plate induced loading presented here more closely resembles the latter. Exploring other load cases combined with a comparison to experimental loading will be the topic of future work. 
To create the detailed subject-specific 3D geometries, high resolution MRI data were required in the current study. MRI is the gold standard for high resolution soft tissue imaging and is feasible for basic research, such as that presented here. Ultrasound imaging may in the future present a more practical approach than MRI. For example, Atkin et al. 2016 [1] recently demonstrated the feasibility of using ultrasound for assessment of geometrical features such as tissue thickness and radius of the ischial tuberosities.

In order to record MRI data for the unloaded geometry, a special support cushion was put in place to help avoid contact between the buttock region and the MRI bedding. However, some subjects may have partially touched the bedding during imaging creating a mildly loaded state and an initially flat buttock surface in the reconstructed geometries.

This is the first study to demonstrate that geometric differences alone can be responsible for large differences in tissue loading outcomes in 3D FEA. The analysis was made possible through subject-specific FEA of 6 study participants. Generating detailed image-based and subject-specific FEA procedures can be a challenging procedure. In the current study all 6 subject specific models were created in a repeatable and consistent matter by coding the modelling and FEA process. In future work this framework may be used to facilitate the creation of a bank of 3D FEA models allowing for detailed comparison and analysis of tissue loading as a function of subject-specific geometry and constitutive parameters.

\section{Conclusion}

The evaluation of pressure ulcer treatment approaches, and the development of assistive technology, regularly relies on computational modelling of soft tissue loading. In turn, the accuracy of these models relies on the fidelity of both the subject-specific constitutive behaviour as well as the subject-specific geometry. However, 2D and non-subject-specific models remain common. This study investigates the importance of subject-specific and 3D geometry for soft tissue load analysis relevant to the study of pressure ulcers. Detailed MRI derived, 3D, and subject-specific finite element models were created of the pelvis and 
buttock, for three $\mathrm{AB}$ volunteers and three volunteers with $\mathrm{SCl}$. Models were assigned with the same mechanical properties, and were subjected to equivalent compressive loading by a flat plate. A large degree of variation was observed, both within, and across subject groups. Although at compressive forces up to $240 \mathrm{~N}$ (35-45\% of body weight) all subjects presented with maximum shear strains in excess of 0.5 , the strain patterns were found to vary considerably. In some subjects, high maximum shear strains developed quickly and remained more localized to the coccyx while for others, the higher strains developed later and deformations were more distributed. Further, although one $\mathrm{SCl}$ subject appeared most vulnerable of all, the other two subjects with $\mathrm{SCl}$ appeared least vulnerable of all (in terms of tissue volume ratio subjected to high maximum shear strains). This highlights the degree of variability in tissue loading findings caused by the differences in geometry. It is clear from this study that findings obtained from a single subject cannot necessarily be generalised to an entire population. To ensure accurate predictions of tissue loading in computational models, realistic 3D and subject specific geometries are therefore required.

\section{Acknowledgements}

This work was supported by the Canadian Institutes of Health Research (CIHR MOP 123434 Mushahwar).

\section{References}

1. Akins, J.S. et al.: Feasibility of freehand ultrasound to measure anatomical features associated with deep tissue injury risk. Med. Eng. Phys. 38, 9, 839-844 (2016).

2. Derler, S., Gerhardt, L.C.: Tribology of skin: Review and analysis of experimental results for the friction coefficient of human skin. Tribol. Lett. 45, 1, 1-27 (2012).

3. Gefen, A.: The biomechanics of sitting-acquired pressure ulcers in patients with spinal cord injury or lesions. Int. Wound J. 4, 3, 222-31 (2007).

4. Gorecki, C. et al.: Impact of pressure ulcers on quality of life in older patients: a systematic review. J. Am. Geriatr. Soc. 57, 7, 1175-83 (2009).

5. Groves, R. et al.: Quantifying the mechanical properties of human skin to optimise future microneedle device design. Comput. Methods Biomech. Biomed. Engin. 15, 1, 73-82 (2012).

6. Josse, G. et al.: Automatic measurement of epidermal thickness from optical coherence tomography images using a new algorithm. Ski. Res. Technol. 17, 3, 314-319 (2011).

7. Levy, A. et al.: An air-cell-based cushion for pressure ulcer protection remarkably reduces tissue stresses in the seated buttocks with respect to foams: Finite element studies. J. Tissue Viability. 23, 1, 13-23 (2014). 
8. Levy, A. et al.: Simulations of skin and subcutaneous tissue loading in the buttocks while regaining weight-bearing after a push-up in wheelchair users. J. Mech. Behav. Biomed. Mater. 28, 436-47 (2013).

9. Lim, D. et al.: Evaluation of a new sitting concept designed for prevention of pressure ulcer on the buttock using finite element analysis. Med. Biol. Eng. Comput. 45, 11, 1079-1084 (2007).

10. Linder-Ganz, E. et al.: Assessment of mechanical conditions in sub-dermal tissues during sitting: A combined experimental-MRI and finite element approach. J. Biomech. 40, 7, 14431454 (2007).

11. Linder-Ganz, E. et al.: Strains and stresses in sub-dermal tissues of the buttocks are greater in paraplegics than in healthy during sitting. J. Biomech. 41, 3, 567-580 (2008).

12. Loerakker, S. et al.: The effects of deformation, ischemia, and reperfusion on the development of muscle damage during prolonged loading. J. Appl. Physiol. 111, 4, 1168-77 (2011).

13. Van Loocke, M. et al.: A validated model of passive muscle in compression. J. Biomech. 39, 16, 2999-3009 (2006).

14. Maas, S.A. et al.: FEBio: Finite Elements for Biomechanics. J. Biomech. Eng. 134, 1, 11005 (2012).

15. Moerman, K.M.: GIBBON (Hylobates Agilis), http://zenodo.org/record/12214, (2014).

16. Moerman, K.M. et al.: Image Based Model Construction, Boundary Condition Specification and Inverse Fea Control : a Basic Matlab Toolkit for Febio. Proc. 11th Int. Symp. Comput. Methods Biomech. Biomed. Eng. 7-8 (2013).

17. Mogensen, M. et al.: Morphology and epidermal thickness of normal skin imaged by optical coherence tomography. Dermatology. 217, 1, 14-20 (2008).

18. Moore, T.L. et al.: Seventeen-point dermal ultrasound scoring system - A reliable measure of skin thickness in patients with systemic sclerosis. Rheumatology. 42, 12, 1559-1563 (2003).

19. Ní Annaidh, A. et al.: Characterization of the anisotropic mechanical properties of excised human skin. J. Mech. Behav. Biomed. Mater. 5, 1, 139-148 (2012).

20. NPUAP/EPUAP/PPPIA: Prevention and treatment of pressure ulcers: clinical practice guideline. Cambridge Media, Osbrone Park, Western Australia (2014).

21. Oomens, C.W.J. et al.: Pressure Induced Deep Tissue Injury Explained. Ann. Biomed. Eng. 43, 2, 297-305 (2014).

22. Redelings, M.D. et al.: Pressure ulcers: more lethal than we thought? Adv. Skin Wound Care. 18, September 2005, 367-372 (2005).

23. $\mathrm{Si}, \mathrm{H} .:$ TetGen, a Delaunay-Based Quality Tetrahedral Mesh Generator. ACM Trans. Math. Softw. 41, 2, 1-36 (2015).

24. Simo, J.C., Taylor, R.L.: Quasi-incompressible finite elasticity in principal stretches. continuum basis and numerical algorithms. Comput. Methods Appl. Mech. Eng. 85, 3, 273-310 (1991).

25. Sims, a. M. et al.: Elastic and viscoelastic properties of porcine subdermal fat using MRI and inverse FEA. Biomech. Model. Mechanobiol. 9, 6, 703-711 (2010).

26. Sopher, R. et al.: Effects of Intramuscular Fat Infiltration, Scarring, and Spasticity on the Risk for Sitting-Acquired Deep Tissue Injury in Spinal Cord Injury Patients. J. Biomech. Eng. 133, 2, 21011 (2011).

27. Vollmer, J. et al.: Improved Laplacian Smoothing of Noisy Surface Meshes. Comput. Graph. Forum. 18, 3, 131-138 (1999).

28. Wu, G.A., Bogie, K.M.: Not just quantity: Gluteus maximus muscle characteristics in ablebodied and $\mathrm{SCl}$ individuals - Implications for tissue viability. J. Tissue Viability. 22, 3, 74-82 (2013).

29. Young, D.L. et al.: Financial impact of improved pressure ulcer staging in the acute hospital with use of a new tool, the NE1 Wound Assessment Tool. Adv. Skin Wound Care. 25, 4, 15866 (2012). 